\title{
FUZZY DECISION AND CONTROL, THE BAYES CONTEXT.
}

\author{
John H. Painter \\ Department of Electrical Engineering \\ Texas A\&M University \\ College Station, Texas 77843-3128 \\ painter@zadok.tamu.edu
}

\begin{abstract}
This is a highly condensed version of a much longer paper. The long version may be obtained directly from the author. This present short paper gives only partial results, showing how it is that Fuzzy Control may be viewed as a particular kind of stochastic (Bayesian) control.
\end{abstract}

\section{Fuzzy Control.}

Fuzzy Control is attributed to Zadeh [1], and to King and Mamdani ${ }^{[2]}$. Fuzzy control is based on identification of "events" in the system under control, parallel to the way human operators manage systems. It implements "IF/THEN" rules, similar to the operation of expert systems in the form, "IFsystem event/THEN-control input." It models system events through the medium of "membership functions," defined on variables measured in the system under control. Such modeling is completely analogous to Bayes event modeling through conditional probability functions. Control input membership functions are also modeled, in analogy to stochastic control densities.

Fuzzy control proceeds in three stages. First, a measured value of event membership function is used to modify its corresponding (IF/THEN) control membership function, in the step called Inference. Next, all the control membership functions so modified are amalgamated to obtain a single control function, in the step called Combination. Finally, a single, unique control input value is obtained from the modified control density, in the step called Defuzzification.

In fuzzy logic, there are two different representations of set logical intersection and union. One is given by the arithmetic pointwise product of membership functions. The other is by the pointwise $\operatorname{Min}\{\cdot\}$ function. Likewise, the union is represented by either the arithmetic pointwise sum or pointwise $\operatorname{Max}\{\cdot\}$ functions. Bellman and Zadeh $^{[3]}$ early examined both representations.

\section{Bayes Form of Fuzzy Control}

Considering multiple-input, single-output control, define the input sensor variables, output control variable, and events in the following manner:

$\mathrm{z}$ : The numerical control variable, a scalar.

$\underline{\mathrm{x}}_{\mathrm{N}}=\left(\mathrm{x}_{1}, \mathrm{x}_{2}, . ., \mathrm{x}_{\mathrm{N}}\right):$ Sensor measurement vector.

$\left\{A_{i}: i=1,2, . ., M\right\}:$ Family of Event Sets,

defined on $\underline{x}_{\mathbf{N}}$.

Define a probability density on the control variable, $z$, conditioned on the measurement vector, $\underline{x}_{N}$. The control membership function is denoted as $p\left(z \mid \underline{x}_{N}\right)$.

The measured vector, $\underline{x}_{\mathrm{N}}$, lies in an $\mathbf{N}$-dimensional vector space, forming a state space for the system under consideration. Upon this system state space is defined a set of "events," $A_{j}$, being $M$ in number. The total set of events covers, and is a partition of, the state space. These events form the antecedents for the IF/THEN control rules. For example, in an aircraft flight management application, the events are called flight modes, having distinctive names like Final Approach, Land, etc.

These events, $A_{i}$, are embedded in the model for the control membership function (density), using the standard ploy of augmenting the joint density with the Sure Event, defined as the union of the $A_{i}$.

Then, we have that

$$
p\left(z \cap \underline{x}_{N}\right)=p\left(\bigcup_{i=1}^{M}\left(z \bigcap \underline{x}_{N} \cap A_{i}\right)\right)
$$

We define the events, $A_{i}$, to be unique, or, in Bayes terminology, mutually exclusive, even though their subset models may not be quite disjoint. The fact that unique events are so represented, reflects a modeling choice, which injects uncertainty into the model. We say that the overlapping partition 
boundaries are fuzzy. As designers, we will choose the events to be unique and then do our best to model the $A_{i}$ as vector-space subsets.

Equation-(1) then transforms to

$$
p\left(z \cap \underline{x}_{N}\right)=\sum_{i=1}^{M} p\left(z \bigcap \underline{x}_{N} \cap A_{i}\right)
$$

In equation-(2), if an input sensor sample vector, $\underline{x}_{N}$, is observed to have a non-empty intersection with $A_{i}$, we say $A_{i}$ occurs. This decision rule is the basis for testing the antecedent of the IF/THEN control rule.

It is the practice in Fuzzy Control to model the antecedents on single sensor variables, rather than on vectors ( $\mathrm{N}$-tuples). These single-variable models are the familiar membership functions. Thus, decision is based on individual vector components, rather than on $\mathrm{N}$-tuples. Define non-empty subsets, $A_{i j}$, corresponding to the respective projections of $A_{i}$ on the $x_{j}$ axes.

Decision tests are then individual, for the $x_{j}$ falling in the $A_{i j}$ events. This is a sub-optimum test, and it has a performance cost. Testing for $x_{1}$ in $A_{i 1}$ and $x_{2}$ in $A_{i 2}$, separately, treats $A_{i}$ as though it were a rectangle in the state space.

By standard manipulations, we obtain

$$
p\left(z \mid \underline{x}_{N}\right)=\sum_{i=1 j=1}^{M N} p\left(z \mid x_{j} \cap A_{i j}\right) \cdot P\left(A_{i j} \mid x_{j}\right)
$$

where we have restricted the $x_{j}$ to be independent and $p\left(\underline{x}_{N}\right)$ has canceled out of numerator and denominator.

The quantities, $p\left(z \mid x_{j} \cap A_{i j}\right)$ correspond to the fuzzy control membership functions. They give control density, conditional on the event that sensor variables fall in projections of $A_{i}$ on corresponding sensor variable axes. We call these projections "sub-events," or "sub-modes." The quantity, $P\left(A_{i j} \mid x_{j}\right)$, corresponds to the input (or sensor) membership function, which is the posterior probability of sub-event (sub-mode), given corresponding sensor variable.

One last simplification is made in the notation, to arrive at a final formulation. The quantity, $p\left(z \mid x_{j} \cap A_{i j}\right)$ is a control density, conditioned on both event, $i$, and sensor, $j$. Although the mathematics supports dependence on $j$, in practice these control densities are generally a function only of $i$, the identifier of the predefined mode. That is, control is based only on knowledge of mode, and is not differentiated as to which sensor supplied that knowledge. Thus, we will simplify as

$$
p\left(z \mid x_{j} \cap A_{i j}\right) \rightarrow p\left(z \mid A_{i}\right)
$$

and there will be only $M$ of these, rather than $M \cdot N$.

Also, $P\left(A_{i j} \mid x_{j}\right)$ may be notationally changed without changing its meaning, as

$$
P\left(A_{i j} \mid x_{j}\right) \rightarrow P\left(A_{i} \mid x_{j}\right)
$$

and there are still $M \cdot N$ of these. Then, equation-(3) becomes

$$
p\left(z \mid \underline{x}_{N}\right)=\sum_{i=1}^{M} p\left(z \mid A_{i}\right) \cdot \prod_{j=1}^{N} P\left(A_{i} \mid x_{j}\right)
$$

The two-fold internal product, $\mathrm{p}(\cdot) \cdot \Pi \cdot \mathrm{P}(\cdot)$, is the weighting of control membership by the net sensor membership. The $\mathbf{N}$-fold product over the sensors is equivalent to the $\mathbf{N}$-fold intersection over the sensors, for a particular mode-event, where "product" is the soft version of the hard fuzzy intersection, "min". The M-fold sum over the events is equivalent to the $\mathbf{M}$-fold union over the events, where "sum" is the soft version of the hard fuzzy union, "max".

The control density may be defuzzified by the centroid method (which is analogous to the Bayes conditional-mean), if the density is continuous and single-moded. Otherwise, the Bayes $\mathcal{M A P}$ estimate may be used if, for instance, the density is discrete or multi-moded, continuous.

\section{Conclusion}

What the Bayes approach shows is that fuzzy control may be viewed as an ensemble-averaged control, where the average is taken over a set of competing uncertain antecedent events, predefined on the system state space.

\section{References.}

[1] Zadeh, L.A.(1974). A Rationale for Fuzzy Control. Transactions of the ASME, Series G (USA). no. 94. pp. 3-4.

[2] King, P.J. and Mamdani, E.H.(1977); The Application of Fuzzy Control Systems to Industrial Processes; Automatica; vol. 13, pp. 235-242.

[3] Bellman, R.E. and Zadeh, L.A.(1970). Decisionmaking in a Fuzzy Environment. Management Science. no. 4. pp. 141-164. 